\section{Uso do Tamoxifeno no Tratamento da Tiveoidite de Riedel: Relato de Um Caso}

\section{RESUMO}

O tratamento da tireoidite de Riedel (TR) consiste em cirurgia nos casos de fibrose local limitada. Na maioria dos casos, entretanto, necessita-se do uso de agentes antiinflamatórios, como os glicocorticóides ou, nos casos de falha ou recidiva, o tamoxifeno pode ser útil. Relatamos um caso de TR em uma mulher negra de 55 anos, associada a hipotireoidismo e hipoparatireoidismo. Avaliamos o tratamento com tamoxifeno na dose de $20 \mathrm{mg}$ duas vezes ao dia, durante onze meses. Após sessenta dias de tratamento, a paciente não relatava os sintomas compressivos antes apresentados. Entretanto, em um seguimento de onze meses, houve pouca melhora objetiva avaliada por ultrassonografia e tomografia seriados de região cervical. $O$ tamoxifeno pode ser útil na TR, principalmente quando o uso do glicocorticóide é contra-indicado. A duração ideal do uso desta terapia não está definida. (Arq Bras Endocrinol Metab 2004;48/6:903908)

Descritores: Tratamento; Tireoidite de Riedel; Hipotireoidismo; Tamoxifeno

\begin{abstract}
Use of Tamoxifen in the Treatment of Riedel's Thyroiditis: Report of a Case.

Treatment of Riedel's thyroiditis (RT) consists of surgery in cases of local limited fibrosis. In most cases, however, it is required the use of antiinflammatory agents like glucocorticoids or, in those who fail to respond or relapse, tamoxifen can be useful. We report a case of RT in a 55-yearold black woman associated with hypothyroidism and hypoparathyroidism. We evaluated the treatment with tamoxifen, $20 \mathrm{mg}$ twice a day, for eleven months. After sixty days of therapy, patient had no compressive symptoms previously presented. However, in a followup of eleven months, there was little objective improvement by regular ultrasonography and computed tomography of the cervical region. Tamoxifen can be useful in RT, mainly when glucocorticoids are not indicated. The ideal duration of this therapy remains to be established. (Arq Bras Endocrinol Metab 2004;48/6:903-908)
\end{abstract}

Keywords: Treatment; Riedel's thyroiditis; Hypothyroidism; Tamoxifen

A TIREOIDITE DE RIEDEL (TR) é uma doença inflamatória crônica da tireóide, reconhecida por Bernhard Riedel em 1883, que posteriormente publicou, em 1896, a descrição de dois pacientes com comprometimento traqueal compressivo secundário à tireóide fibrótica (1). Possíveis lesões associadas são a fibrose retroperitoneal e mediastinal, a colangite esclerosante, o pseudo-tumor orbitário e o envolvimento de parótidas (2,3). Estima-se que, em 34\% dos casos de TR, ao menos uma das lesões citadas acima esteja presente no mesmo paciente (4). A etiologia da TR não está totalmente apresentação de casos

\author{
Marcos Sadao Iwakura \\ Rosita Fontes
} Instituto Estadual de Diabetes e
Endocrinologia - IEDE, Rio de
Janeiro, RJ. 
definida. Fatores parácrinos liberados localmente por macrófagos, eosinófilos e linfócitos infiltrados agiriam como estimuladores do processo fibrogênico $(2,5)$. Os eosinófilos seriam capazes de estimular a proliferação fibroblástica liberando e expressando genes para citocinas e conseqüente proliferação fibroblástica $(2,6)$. A importância do tratamento da TR se baseia no fato de que, em geral, se não tratada, ocorre lenta e progressiva piora da lesão (7). Tratamento cirúrgico definitivo não é recomendado, pois uma ressecção completa da lesão pode causar danos pela extensão da fibrose extratireoideana (1,7-9). No tratamento clínico, os glicocorticóides são as drogas de escolha, baseando-se em vários relatos sugerindo diminuição do bócio com o uso de prednisona (1,7). Há relatos de sucesso terapêutico com o uso de tamoxifeno - 20 a $40 \mathrm{mg} /$ dia em casos de resistência ao corticóide (9), recorrência da doença (10) ou, ainda, em associação no início do tratamento com metilprednisolona (11).

\section{RELATO de CASO}

Paciente APS, sexo feminino, 55 anos, negra, do lar, nascida e residente no Rio de Janeiro, RJ. Queixava-se, há um ano, de tosse seca, aumento de volume em região cervical anterior e ocasionais disfagia e dispnéia. Constatado um bócio volumoso, programou-se tireoidectomia. A cirurgia não foi realizada em virtude da impossibilidade técnica de ressecção da glândula. A biópsia a céu aberto realizada na ocasião demonstrou permeação inflamatória predominantemente linfoplasmocitária com freqüentes eosinófilos, além de esparso tecido muscular estriado dissociado por fibrose colágena freqüente, compatível com tireoidite de Riedel. Nesta ocasião, avaliada no ambulatório de Tireóide do Instituto Estadual de Diabetes e Endocrinologia IEDE/RJ, a paciente não apresentava outros dados positivos à anamnese além dos sintomas compressivos pelo bócio. Tinha antecedentes de hipertensão arterial e intolerância à glicose. Não relatava história familiar de tireoidopatias. Fazia uso contínuo de Propranolol $80 \mathrm{mg} /$ dia e enalapril $10 \mathrm{mg} / \mathrm{d}$. Ao exame físico, encontrava-se eupnéica, corada, fácies atípica. Pressão arterial $=160 / 100 \mathrm{mmHg}$, pulso regular $=80$ batimentos por minuto, índice de massa corpórea $=34,9 \mathrm{~kg} / \mathrm{cm}^{2}$. Em região cervical, palpava-se uma tireóide de volume aumentado, com consistência pétrea, indolor e superfície irregular sem nódulos delimitados, medindo, no maior diâmetro de cada lobo, $9 \mathrm{~cm}$ (lobo direito) e 7,5cm (lobo esquerdo). $\mathrm{O}$ istmo era de difícil delimitação. Ausência de linfadenomegalias. As auscultas cardíaca e pulmonar eram normais e não havia alterações no abdômen . Os reflexos profundos eram normorreativos e não havia edema local ou generalizado.

Os exames laboratoriais iniciais foram: hemoglobina $=10,3$ (VR: 11-16,5); hematócrito $=32,3 \%$ $(\mathrm{VR}=35-50 \%)$; velocidade de hemossedimentacão= $100 \mathrm{~mm} /$ hora; coagulograma normal, glicemia $=$ $103 \mathrm{mg} / \mathrm{dL}$; sódio $=139 \mathrm{mMol} / \mathrm{L}$ (VR: 136-146); potássio $=4,0 \mathrm{mMol} / \mathrm{L}$ (VR: 3,5-5,1); TSH Quimioluminescência $(\mathrm{Ql})=19,3 \mathrm{mcU} / \mathrm{mL}(\mathrm{VR}: 0,4-$ $4,0)$; tiroxina livre estimada $(\mathrm{Ql})=0,8 \mathrm{ng} / \mathrm{dL}$ (VR: 0,8 1,9); anti-tireoperoxidase $(\mathrm{Ql})=27 \mathrm{UI} / \mathrm{ml}$ (VR: até 35$)$. A tomografia de região cervical mostrava tireóide com aumento difuso, compressão de traquéia, difícil definição de estruturas vasculares adjacentes e ausência de plano de clivagem definido com o músculo esternoclidomastóideo. Os exames tomográficos de mediastino, abdômen e órbitas não evidenciaram alterações. A ultrassonografia de tireóide (julho/2002) estimava o volume do lobo direito em $51,8 \mathrm{ml}$ e do lobo esquerdo em $57,6 \mathrm{ml}$. Não evidenciava nódulos. Em novembro de 2002, foi iniciado tratamento com tamoxifeno $20 \mathrm{mg} 2 \mathrm{x} / \mathrm{d}$. Mantivemos a reposição com levotiroxina $125 \mathrm{mcg} /$ dia, iniciada há cerca de quatro meses. Após um mês de uso do tamoxifeno, houve melhora subjetiva dos sintomas compressivos pelo bócio. A paciente encontrava-se assintomática com boa tolerância à droga. Não relatava fogachos ou sangramento vaginal. Em fevereiro de 2003, em eutireoidismo - TSH $(\mathrm{Ql})=2,4 \mathrm{mcU} / \mathrm{mL}$-, a paciente queixava-se de parestesias em face e membros superiores, apresentava sinais de Trousseau e Chvostek positivos, e os exames complementares confirmaram hipoparatireoidismo: cálcio iônico $=3,3 \mathrm{mg} / \mathrm{dL}$ (VR: 4,5-5,2); cálcio total $=7,0 \mathrm{mg} / \mathrm{dL}$ (VR: 8,4-9,7); fósforo $=8,4 \mathrm{mg} / \mathrm{dL}$ (VR: 2,7-4,5); paratormônio - molécula intacta $(\mathrm{Ql})=$ 10,2pg/ml (VR: 7-53). Foi prescrita, na época, vitamina D3, dez gotas/dia (1 gota $=4590 \mathrm{UI})$ e carbonato de cálcio por via oral $\mathrm{lg} /$ dia. Aos seis meses de uso do tamoxifeno, uma nova ultrassonografia de tireóide (maio de 2003) demonstrou diminuição do bócio com estimativa de medidas para o lobo direito de $6,2 \times 3,7 \times$ $3,5 \mathrm{~cm}$ (volume $=41,7 \mathrm{ml})$ e para o lobo esquerdo de 6,6 x 3,6 x $3,7 \mathrm{~cm}$ (volume $=45,7 \mathrm{ml}$ ). A dose de $40 \mathrm{mg} / \mathrm{dia}$ de tamoxifeno foi mantida. Em reavaliação em outubro de 2003, completaram-se onze meses de uso do antiestrogênio. Apesar da paciente ter permanecido assintomática em relação aos sintomas compressivos pelo bócio, os exames ultrassonográfico e tomográfico de controle para avaliação da extensão da fibrose não evidenciaram redução posterior significativa do tamanho da lesão. Mantivemos a reposição de hormônio tireoideano, cálcio e vitamina $\mathrm{D}$, além do tamoxifeno na dose de $40 \mathrm{mg} /$ dia. Houve boa tolerância ao 
antiestrogênio pela paciente, não tendo ocorridos sinais de hiperplasia endometrial, inclusive por avaliação ultrassonográfica transvaginal periódica.

\section{DISCUSSĀO}

A TR é uma doença fibroinflamatória crônica da tireóide caracterizada por fibrose densa e invasiva, acometendo a glândula e seu tecido circundante (7). A prevalência é maior no sexo feminino, sendo a relação com o sexo masculino de $4: 1$. A doença ocorre mais entre os 30 e 50 anos $(7,9)$. Há relatos de que a incidência desta doença vem diminuindo, embora o número de casos relatados na literatura não reflitam necessariamente os casos vistos clinicamente (12).

A etiologia desta patologia permanece controversa. Evidências sugerem que a TR possa ser uma manifestação local de uma doença sistêmica fibrosante $(5,7)$ ou uma lesão fibrótica primária $(8)$. Um mecanismo auto-imune é sugerido pela detecção de auto-anticorpos contra a tireóide, resposta terapêutica aos corticosteróides e a presença de infiltrado celular mononuclear na lesão $(5,7)$. Heufelder e cols. (1996) demonstraram, através de técnicas de imuno-histoquímica, a presença de infiltração eosinofílica em 15 de 16 amostras analisadas de biópsia compatível com TR. Em amostras de tecidos de tireóide normal ou com outras patologias, não foi evidenciada eosinofilia de tal magnitude (6). Relatos sugerem que extratos de eosinófilos são capazes de estimular a fibrogênese em meios condicio-nados, liberando e expressando genes para citocinas como o fator transformador de crescimento beta (TGF-B), o qual, sob certas circunstâncias, poderia inibir ou, neste caso, estimular a proliferação e a síntese fibroblástica (2). O conceito de que a TR seria uma forma tardia ou uma variante da tireoidite de Hashimoto $(\mathrm{TH})$ ou, ainda, uma forma da tireoidite sub-aguda granulomatosa foi abandonado pela história natural destas $(4,13)$. A predisposição genética para a TR foi sugerida após a descrição de dois irmãos, filhos de pais consangüíneos, os quais desenvolveram fibrose multissistêmica, estando presente a TR em um deles. Casos familiares de TR isolada não foram, ainda, descritos (14).

Clinicamente, os pacientes se apresentam com aumento recente de um bócio pré-existente tipicamente indolor. Sintomas de compressão local, como estridor, rouquidão, sensação de sufocação, dispnéia e disfagia, podem estar presentes $(7,15)$. Bradicardia e síncope denotam compressão ou invasão do nervo vago (7). Ao exame da tireóide, percebe-se bócio de dimensões variadas, com acometimento bilobar. A consistência pétrea é característica e pode haver confusão inicial com linfoma da tireóide, carcinoma anaplásico da tireóide ou sarcoma (9). Os linfonodos regionais não são acometidos (12).

Em relação à função tireoideana, a maioria dos casos mantém-se em eutireoidismo, sendo as taxas de prevalência para o hipotireoidismo variáveis, entre 25 a $32 \%$ dos pacientes (7). Esta disfunção resulta do envolvimento completo da tireóide e, em alguns casos, a associação com TH tem sido implicada $(1,3)$. A positividade dos anticorpos antitireoideanos oscila entre $40-70 \%$ dos casos (7). O caso descrito evoluiu com hipotireoidismo durante a progressão do bócio, não havendo aparente doença auto-imune concomitante, evidenciada pela ausência de anticorpos antitireoideanos em duas dosagens séricas. A apresentação com hipoparatireoidismo é rara, mas tem sido relatada quando há fibrose extensa. Infiltração gradual fibrótica das glândulas é o provável mecanismo etiológico, além do comprometimento vascular com isquemia local. Há descrições de casos de recuperação funcional das paratireóides com a regressão do bócio após tratamento cirúrgico (16) ou após uso de glicocorticóides (1). Nossa paciente apresentou hipoparatireoidismo espontâneo provavelmente pela extensão da fibrose. O quadro foi evidenciado por níveis séricos elevados de fósforo e diminuídos de cálcio associados a níveis inapropriadamente baixos de paratormônio (PTH). As duas dosagens do PTH (Ql) foram de 10,2 e 12,0pg/ml (VR: 7-53). Provavelmente trata-se de um hipoparatireoidismo permanente, estando a paciente dependente de tratamento específico de reposição há mais de seis meses. Com o uso de vitamina D3 e cálcio via oral, houve melhora em relação aos sintomas de hipocalcemia, elevação do cálcio e redução do fósforo séricos.

Há relatos da associação entre TR e outras lesões fibrosantes em mediastino, retroperitôneo, órbitas e ducto colédoco (12). Não encontramos tais lesões na paciente descrita, avaliada por estudo tomográfico nestes sítios.

Além dos dados já citados, outros podem contribuir para o diagnóstico da TR. A velocidade de hemossedimentação pode estar normal ou elevada. Anticorpos antitireoideanos - anti-peroxidase e antitireoglobulina - podem estar presentes em 40-70\% dos casos $(7,12)$. A punção aspirativa de tireóide para biópsia, em geral, não é elucidativa, além da dificuldade técnica para a sua realização em virtude da consistência do bócio, sendo recomendada a biópsia a céu aberto. Os exames de imagem são inespecíficos: a cintilografia de tireóide mostra captação diminuída e heterogênea; a ultrassonografia de tireóide, em geral, evidencia áreas hipoecóicas 
devido à fibrose; a tomografia computa-dorizada revela melhor a extensão da lesão e a tireóide se mostra hipodensa ou normal $(7,12)$.

$\mathrm{O}$ diagnóstico definitivo da TR é histopatológico e baseia-se nos critérios de Woolner (1957), os quais foram modificados por Schwaregerle em 1988: processo inflamatório fibrótico grosseiro visível envolvendo a tireóide; evidência grosseira ou histológica de extensão para estruturas adjacentes; ausência de reação granulomatosa e ausência de neoplasia (12). À microscopia, há tecido fibroso abundante, matriz hialinizada, ausência de células gigantes, presença de células inflamatórias - linfócitos $\mathrm{B}$ e $\mathrm{T}$, polimorfonucleares (predominância dos eosinófilos), plasmócitos e macrófagos -, e folículos tireoideanos degenerados ou ausentes (3).

$\mathrm{O}$ principal diagnóstico diferencial se faz com a variante fibrosa da $\mathrm{TH}$ (12). Neste último, diferentemente da TR, há menos tecido fibroso, infiltrado linfóide abundante, não há associação com fibrose extracervical ou invasão local, a cápsula tireoideana encontra-se íntegra, não há alteração vascular e a metaplasia oncocítica é mais extensa (3). O diagnóstico definitivo no caso descrito foi realizado por biópsia a céu aberto. O diferencial com a $\mathrm{TH}$ foi estabelecido pela grande extensão de fibrose local, inclusive com invasão de áreas adjacentes, como músculos e vasos. Outros dados sugestivos para TR foram os achados microscópicos típicos, tecido conjuntivo hialinizado local e ausência de anticorpos antitireoideanos.

O tratamento da TR é baseado em casos isolados relatados na literatura, não havendo consenso quanto à terapia inicial ideal ou, ainda, estudos clínicos evidenciando real superioridade de uma das modalidades terapêuticas. Quando há envolvimento lobar bilateral associado a sintomas de compressão traqueal, recomendase ressecção profunda do istmo para alívio local, podendo ser realizada concomitantemente à biópsia cirúrgica para confirmação histopatológica (1). No entanto, uma ressecção completa da lesão pode causar danos indesejáveis pela extensão da fibrose extratireoideana (1,7-9). Houve insucesso na tentativa inicial de tireoidectomia em nossa paciente, previamente ao diagnóstico de TR, em razão da impossibilidade de delimitação da lesão em relação às estruturas próximas locais.

No tratamento clínico, os glicocorticóides são as drogas de escolha, baseando-se em vários relatos sugerindo diminuição do bócio com o uso de prednisona $(1,7)$. Lo e cols. (1998) relataram um caso de TR, o qual, após introdução de corticoterapia depois de um mês do início da sintomatologia, evoluiu com regressão do volume do bócio (1). Altas doses de corticóide $(80-100 \mathrm{mg} / \mathrm{d}$ de prednisona ou equivalente) parecem ser críticas no início do tratamento, embora doses de manutenção (5$10 \mathrm{mg} / \mathrm{d}$ ) mostrem-se suficientes a longo prazo na maioria dos casos $(1,2,4,8)$. O mecanismo de ação proposto dos glicocorticóides consiste na redução da produção de citocinas (1). A terapia imunossupressora com ciclofosfamida foi relatada com resultado satisfatório, apesar dos efeitos adversos descritos (2). A levotiroxina pode ser usada com o propósito de reposição hormonal nos casos que evoluem com hipotireoidismo ou para supressão tireoideana (2). Entretanto, tanto o grau pelo qual o TSH exacerbaria o processo inflamatório quanto seu papel na manutenção da doença em remissão quando em níveis suprimidos, não estão totalmente elucidados (1).

O tamoxifeno, um antiestrogênio nãoesteróide, foi aprovado para uso clínico no câncer de mama em mulheres pós-menopausa na década de 70 $(17,18)$. A ação dos antiestrogênios ocorre pela ligação a sítios do receptor do estradiol de forma competitiva, podendo, ainda, interferir na função dos fatores coativadores no processo de transcrição gênica $(19,20)$. Embora considerado um antiestrógeno, pode estimular o endométrio com conseqüente risco três a quatro vezes maior de câncer endometrial em mulheres pós-menopausa (21). A observação de que o tamoxifeno apresenta bons resultados terapêuticos em casos de câncer de mama receptor-estrogênico negativos, permitiu a conclusão de que outro mecanismo de ação da droga estaria envolvido no processo antitumoral. Inicialmente in vitro, demonstrou-se que o tamoxifeno induz a secreção autócrina em fibroblastos humanos fetais desprovidos de receptores estrogênicos, do fator de transformação de crescimento beta (TGF-beta). Este pertence a uma família de três peptídeos regulatórios multifuncionais com importante função inibitória no controle de proliferação e diferenciação da maioria dos tecidos humanos epiteliais, incluindo células normais e tumorais (22). Posteriormente, Butta e cols. (1992) demonstraram a indução de TGF-beta extracelular no estroma de tumores de mama humanos após três meses de uso do tamoxifeno, implicando este peptídeo como um potencial mediador na ação terapêutica desta droga (23). Outros mecanismos de ação antiproliferativa propostos para o tamoxifeno são o antagonismo à calmodulina, promovendo redução intracelular de cálcio, e outros processos dependentes da proteína C quinase $(24,25)$.

Relatos prévios de boa resposta ao tamoxifeno no tratamento de tumores benignos 
fibróticos, como os desmóides e a fibrose retroperitoneal (26), objetivaram a avaliação desta medicação no tratamento da TR. Em uma série inicial de quatro pacientes com TR sintomática e progressiva, apesar do tratamento prévio com glicocorticóides e cirurgia, o tamoxifeno resultou em melhora sintomática, redução e até desaparecimento do processo fibrótico em um caso (9). Jaap e cols. (2002) demonstraram sucesso com o uso do tamoxifeno em uma paciente com TR apresentando recorrência da doença após retirada do glicocorticóide. Houve melhora lenta e progressiva dos sintomas compressivos, assim como redução do bócio num curso de seis meses de terapia (10). Dabelic e cols. (2003), de forma semelhante, constataram melhora subjetiva e objetiva - método ultrassonográfico - em uma paciente de 46 anos de idade com TR, após oito meses de tratamento com tamoxifeno na dose de $10 \mathrm{mg}$ ao dia. Neste caso, a metilprednisolona já havia sido utilizada sem sucesso (11). Admite-se, atualmente, que a efetividade do uso do tamoxifeno no tratamento da TR e de outras doenças fibróticas possa estar relacionada ao estímulo da produção de TGF-beta, sendo este um potente inibidor do crescimento de fibroblastos e de células epiteliais (7-11,22,26). Dentre os possíveis efeitos adversos relacionados ao uso do tamoxifeno, relacionam-se os fogachos, a irregularidade menstrual e a hiperplasia endometrial associada a risco aumentado para câncer de endométrio $(17,19)$. Iniciamos, em nossa paciente, o tratamento com tamoxifeno na dose de $40 \mathrm{mg} /$ dia sem uso prévio de corticoterapia. A escolha do antiestrogênio baseou-se nos relatos anteriores de melhora objetiva da lesão com esta medicação. Além disso, a corticoterapia prolongada em dose alta não é isenta de efeitos adversos (27).

Tratando-se de uma paciente obesa, hipertensa e intolerante à glicose, optamos por não utilizar de início o corticóide. Relatamos o caso de uma paciente com TR complicada com hipotireoidismo não autoimune e hipoparatireoidismo. Não houve evidências de outras lesões fibroescleróticas.

Durante o tratamento por onze meses com tamoxifeno, apesar da melhora subjetiva importante, evidenciamos apenas redução inicial e posterior estabilização da lesão tireoideana. Em conclusão, o tratamento da TR com tamoxifeno pode ser considerado uma opção aos pacientes com contra-indicação relativa aos glicocorticóides, não havendo definição quanto ao tempo de uso ideal do antiestrogênio. São necessários relatos de novos casos para se avaliar o melhor tratamento desta doença, a qual, apesar de incomum, pode apresentar importante morbidade.

\section{REFERÊNCIAS}

1. Lo JC, Loh K, Rubin AL, Cha I, Greenspan FS. Riedel's thyroiditis presenting with hypothyroidism and hypoparathyroidism: dramatic response to glucocorticoid and thyroxin therapy. Clin Endocrinol 1998;48:815-8.

2. Owen $\mathrm{K}$, Lane $\mathrm{H}$, Jones $\mathrm{K}$. Multifocal fibroesclerosis: a case of thyroiditis and bilateral lachrymal gland involvement. Thyroid 2001;11(12):1187-90.

3. Baloch ZW, Saberi M, Livolsi VA. Simultaneous involvement of thyroid by Riedel's disease and fibrosing Hashimoto's thyroiditis: a case report. Thyroid 1998;8(4):337-41.

4. Natt N, Heufelder AE, Hay ID, Grant CS, Goellner JR. Extracervical fibrosclerosis causing obstruction of a ventricular-peritoneal shunt in a patient with hydrocephalus and invasive fibrous thyroiditis (Riedel's struma). Clin Endocrinol 1997;47:107-11.

5. Heufelder AE, Hay ID. Further evidence for autoimmune mechanisms in the pathogenesis of Riedel's invasive fibrous thyroiditis. J Intern Med 1995;238:85-6.

6. Heufelder AE, Goellner JR, Bahn RS, Gleich GJ, Hay ID. Tissue eosinophilia and eosinophil degranulation in Riedel's invasive fibrous thyroiditis. J Clin Endocrinol Metab 1996;81(3):977-84.

7. Yasmeen T, Khan S, Patel SG, Reeves WA, et al. Riedel's thyroiditis: report of a case complicated by spontaneous hypoparathyroidism, recurrent laryngeal nerve injury, and Horner's syndrome. J Clin Endocrinol Metab 2002;87(8):3543-7.

8. Zimmermann-Belsing T, Feudt-Rasmussen U. Riedel's thyroiditis: an autoimmune or primary fibrotic disease? J Intern Med 1994;235:271-4.

9. Few J, Thompson NW, Angelos P, Simeone D, Giordano T, Reeve T. Riedel's thyroiditis: treatment with Tamoxifen. Surgery 1996;120(6):993-9.

10. Jaap MD, Dempster J. Tamoxifen therapy in steroidresistant Riedel's disease. Scot Med J 2002;47(1):12-3.

11. Dabelic N, Jukic T, Labar Z, Novosel SA, Matesa N, Kusic Z. Riedel's thyroiditis treated with Tamoxifen. Croat Med J 2003;44:239-41.

12. Schwaegerle SM, Bauer TW, Esselstyn CB. Riedel's thyroiditis. Am J Clin Pathol 1988;90:715-22.

13. Armigliato M, Paolini R, Bianchini E, Monesi G, Zamboni S, D'Andrea E. Hashimoto's thyroiditis and Graves' disease associated with retroperitoneal fibrosis. Thyroid 2002; 12(9):829-31.

14. Best TB, Munro RE, Burwell S, Volpé R. Riedel's thyroiditis associated with Hashimoto's thyroiditis, hypoparathyroidism, and retroperitoneal fibrosis. J Endocrinol Invest 1991;14:767-72.

15. Frankenthaler R, Batsakis JG, Suarez PA. Tumefactive fibroinflamatory lesions of the head and neck. Ann Otol Rhinol Laryngol 1993;102:481-2. 
16. vChopra D, Wool MS, Crosson A, Sawin CT. Riedel's struma associated with subacute thyroiditis, hypothyroidism, and hypoparathyroidism. J Clin Endocrinol Metab 1978;46(6):869-71.

17. Jordan VC, Morrow M. Tamoxifen, Raloxifen, and the prevention of breast cancer. Endocr Rev 1999;20(3):253-78.

18. Khovidhunkit W, Shoback DM. Clinical effects of Raloxifene Hydrochloride in women. Ann Intern Med 1999;130(5):431-9.

19. Santen RJ. Long term Tamoxifen therapy: can an antagonist become an agonist? J Clin Endocrinol Metab 1996;81(6):2027-30.

20. Koseki Y, Zava DT, Chmness GC, McGuire WL. Estrogen receptor translocation and replenishment by the antiestrogen Tamoxifen. Endocrinology 1977;101(4):1104-10.

21. Grey AB, Stapleton JP, Evans MC, Reid IR. The effect of the antiestrogen Tamoxifen on cardiovascular risk factors in normal postmenopausal women. J Clin Endocrinol Metab 1995;80(11):3191-5.

22. Arteaga CL, Tandon AK, von Hoff DD, Osborne CK. Transforming growth factor beta: potential autocrine growth inhibitor of estrogen receptor negative human breast cancer cells. Cancer Res 1988;48:3898-904.

23. Butta A, MacLennan K, Flanders KC, Sacks NPM, Smith I, McKinna $A$, et al. Induction of transforming growth fac- tor beta in human breast cancer in vivo following Tamoxifen treatment. Cancer Res 1992;52:4261-4.

24. Fanidi A, Courion-Guichardaz C, Fayard J, Pageaux J, Laugier C. Effects of Tamoxifen, Tamoxifen metabolites, and nafoxidine on adenosine $3^{\prime} 5$ ' monophosphate phosphodiesterase: correlations with growth inhibitory activities but not estrogen receptor affinities. Endocrinology 1989;125(3): 1187-93.

25. Hoelting $\mathrm{T}$, Siperstein A, Duh $\mathrm{Q}$, Clark $\mathrm{OH}$. Tamoxifen inhibits growth, migration, and invasion of human follicular and papillary thyroid cancer cells in vitro and in vivo. J Clin Endocrinol Metab 1995;80(1):308-13.

26. Clark CP, Vanderpool D, Preskitt JT. The response of retroperitoneal fibrosis to Tamoxifen. Surgery 1991;109(4):502-6.

27. Chrousos GR. Glucocorticoid therapy. In: Felig $P$, Frohman LA. Endocrinology and Metabolism. $4^{\text {th }}$ edition. McGraw Hill, USA, 2001. p. 609-14.

Endereço para correspondência:

Marcos Sadao Iwakura

Rua Foz do Iguaçu 711

86061-000 Londrina, PR

E-mail: marcossadao1@yahoo.com.br 